\title{
Factores que influyen en la aceptación de la plataforma de enseñanza-aprendizaje Nexus en la Facultad de Filosofía y Letras de la UANL (Factors that influence the acceptance of the learning management system in the School of Philosophy and Arts at UANL)
}

\author{
Rubén Suárez Escalona \\ Armando Tijerina García* \\ Gloria Nelly Salas Celestino* \\ María de la Luz Escalona Galindo*
}

\begin{abstract}
It is indisputable that technology advances exponentially, and people try to adapt to change constantly, however, the change is so fast that many times users can get uncomfortable with the new technology and generate resistance to its use. For this reason, the intention of this study is to propose a model of acceptance of educational modifying the TAM (Technology Acceptance Model) to the needs of the educational context, by adding the variable perception of playful. The study was conducted at the Faculty of Philosophy and Letters of the UANL, where 100 surveys were applied to undergraduate students, obtaining a good reliability in the designed instrument. Later, the statistical package, SPSS (Statistical Package for the Social Sciences) was used to analyze the data, using the method of successive steps of the multivariate regression. The results of the study show that there are significant relationships between the variables in he proposed model. In general, the study shows in a clear and parsimonious way the most important factors that influence the acceptance of the Nexus platform, therefore, it is concluded that it can be applied in the acceptance of educational technology.
\end{abstract}

Key words: e-learning, learning management system (LMS), Task Fit Technology (TTF), Technology Acceptance Model (TAM), Unified Theory of Acceptance and Use of Technology (UTAUT)

JEL: M15, 032, 033.

- Universidad Autónoma de Nuevo León, Facultad de Contaduría Pública y Administración, Centro de Desarrollo Empresarial y Posgrado, San Nicolás de los Garza, N.L. México.Email: ruben.suarezes@uanl.edu.mx

* Universidad Autónoma de Nuevo León, Facultad de Contaduría Pública y Administración, Centro de Desarrollo Empresarial y Posgrado, San Nicolás de los Garza, N.L. México. Email: ar_tijerina@hotmail.com

- Universidad Autónoma de Nuevo León, Facultad de Ingeniería Mecánica y Eléctrica, San Nicolás de los Garza, N.L. México. Email:ng_gloria_nelly@hotmail.com

* Universidad Autónoma de Nuevo León, Facultad de Psicología, Monterrey, N.L. México. Email: maluzescalona@hotmail.com 
Resumen. Es indiscutible que la tecnología avanza de manera exponencial, las personas intentan adaptarse al cambio frecuente y constante, sin embargo, el cambio es tan rápido que muchas veces los usuarios pueden llegar a sentirse incómodos con la nueva tecnología y generar una resistencia a su uso. Por tal motivo, la intención de este estudio es proponer un modelo de aceptación de tecnología educativa, modificando el modelo TAM por sus siglas en inglés (Technology Acceptance Model) a las necesidades del contexto educativo, mediante la adición de la variable percepción de lúdica. El estudio se realizó en la Facultad de Filosofía y Letras de la UANL, en donde se aplicaron 100 encuestas a estudiantes de licenciatura, obteniendo una buena confiabilidad en el instrumento diseñado, posteriormente se utilizó el paquete estadístico, SPSS por sus siglas en inglés (Statistical Package for the Social Sciences) para analizar los datos, mediante el método de pasos sucesivos de la regresión multivariada. Los resultados del estudio muestran que existen relaciones significativas entre las variables que se plantean en el modelo propuesto, y el estudio muestra de una manera clara y parsimoniosa los factores más importantes que influyen en la aceptación de la plataforma Nexus, por lo anterior, se concluye que se puede aplicar en la aceptación de tecnología educativa, en general.

Palabras clave: educación a distancia, Modelo Ajuste Tarea-Tecnología (TTF), Modelo de Aceptación de Tecnología (TAM), plataformas de educación a distancia, Teoria Unificada de aceptación de Tecnología (UTAUT)

\section{Introducción}

El crecimiento de la tecnología impacta en todas las áreas del conocimiento, los profesionistas buscan mantenerse actualizados para seguir siendo competitivos. Las nuevas tecnologías de la Información y comunicación (TIC), han llegado a romper paradigmas, lo que se consideraba como imposible, ahora se considera como lo que no ha sido posible hasta el momento, esto deja abierta una ventana de posibilidades. Sin embargo, la aceptación de las nuevas tecnologías no siempre es aceptada con éxito.

Un ejemplo de esto lo podemos ver en el sector educativo, en donde la matrícula en educación a distancia $(\mathrm{EaD})$ por medio de plataformas tecnológicas tiene poca afluencia en la Universidad Autónoma de Nuevo León (UANL), según la Asociación Nacional de Universidades e Instituciones de Educación Superior (ANUIES, 2017) la UANL cuenta con una matrícula en la modalidad no escolarizada de 20,401, lo que lo ubica en el lugar número nueve, con respecto a otros estados de la república, cabe mencionar que de 
los 20,401 estudiantes solo 400 pertenecen a la UANL, el resto son de universidades privadas.

Tabla 1. Estudiantes matriculados en modalidad no escolarizada

\begin{tabular}{llllll}
\hline Lugar & $\begin{array}{l}\text { Entidad } \\
\text { Federativa }\end{array}$ & $\begin{array}{l}\text { Alumnos } \\
\text { matriculados }\end{array}$ & Lugar & $\begin{array}{l}\text { Entidad } \\
\text { Federativa }\end{array}$ & $\begin{array}{l}\text { Alumnos } \\
\text { matriculados }\end{array}$ \\
\hline 1 & Ciudad de México & 202,891 & 17 & Guerrero & 5,925 \\
2 & Veracruz & 53,523 & 18 & Colima & 5,640 \\
3 & México & 45,204 & 19 & Hidalgo & 5,252 \\
4 & Chiapas & 33,576 & 20 & Oaxaca & 5,224 \\
5 & Puebla & 28,242 & 21 & Chihuahua & 4,479 \\
6 & Jalisco & 25,184 & 22 & Durango & 4,424 \\
7 & Guanajuato & 21,981 & 23 & Morelos & 4,339 \\
8 & Sinaloa & 20,893 & 24 & Quintana Roo & 4,202 \\
9 & Nuevo León & 20,401 & 25 & Yucatán & 2,870 \\
10 & Michoacán & 10,935 & 26 & Zacatecas & 2,821 \\
11 & Nayarit & 10,635 & 27 & Aguascalientes & 2,658 \\
12 & San Luis Potosí & 10,173 & 28 & Campeche & 1,262 \\
13 & Tabasco & 9,893 & 29 & Tamaulipas & 1,167 \\
14 & Baja California & 9,184 & 30 & Tlaxcala & 972 \\
15 & Querétaro & 7,823 & 31 & Baja California Sur & 561 \\
16 & Coahuila & 7,435 & & & \\
\hline
\end{tabular}

Fuente: Anuario de educación superior 2016-2017 (ANUIES, 2017).

Para impulsar la EaD, la UANL desarrollo una plataforma en función de sus necesidades llamada Nexus, la cual ha ido mejorando su funcionalidad en cada nueva versión. Sin embargo, aún existe resistencia a su uso, por parte de la población universitaria. Por tal motivo, la presente investigación tiene como objetivo identificar los factores que influyen en la aceptación de la plataforma de Enseñanza-Aprendizaje (E-A) Nexus.

El modelo TAM, fue desarrollado por Davis (1989), con el objetivo de crear instrumentos de medición eficientes para la aceptación de tecnología. Desde su creación hace más de un cuarto de siglo, se ha publicado una gran cantidad de artículos basándose en el modelo TAM y en adaptaciones subsecuentes. (Marangunić y Granić, 2015) 
Sin embargo, durante la revisión de literatura no se encontraron artículos en los que se utilizara el modelo TAM, para medir la aceptación de la plataforma Nexus, que es la plataforma oficial de la UANL. Por otra parte, los modelos propuestos en los estudios empíricos no consideran la lúdica como un factor causal en la aceptación de la tecnología.

A manera de justificación, el presente estudio permitirá identificar los factores más importantes en la aceptación de la plataforma Nexus, permitiendo implementar acciones para mejorar dichos factores, y esto a su vez permita, que la educación pueda llegar a sectores de la población, que no pueden asistir a clases por cuestiones personales. Aunado a esto, la globalización y el crecimiento de internet, exigen una educación continua en los profesionistas egresados, los cuales también se verán beneficiados.

Por todo lo anterior, el propósito de este estudio es probar una modificación al modelo TAM, que relaciona las variables, aceptación de tecnología con las variables percepción de utilidad, percepción de fácil uso y percepción de lúdica.

\section{Marco teórico}

Con base a la teoría psicológica de la acción razonada del inglés (theory of reasoned action) propuesta por Azjen (1985) y de la teoría del comportamiento planeado del inglés (theory of planned behavior), propuesta por Azjen y Fishbein (1980). El modelo TAM adopta dos variables independientes, llamadas percepción de utilidad y percepción de facilidad de uso, que junto con la variable actitud hacia el uso del sistema, pretenden explicar la motivación del usuario hacia la intención de usar el sistema. (Marangunić, \& Granić).

Posteriormente en 2000, Davis en colaboración con Venkatesh crearon el modelo TAM 2, en el cual introducen variables externas como las normas subjetivas, la imagen, la relevancia del trabajo, la calidad de la salida, los resultados demostrables y la experiencia, las cuales influyen sobre la percepción de fácil uso y la percepción de utilidad, haciendo el modelo más extenso tratando de explicar en mayor medida la aceptación de tecnología.

Siguiendo con la línea de tiempo Venkateh, Morris, Davis y Davis, (2003) modifican el modelo TAM 2 y lo renombran con el nombre de UTAUT 
por sus siglas en inglés (The unified theory of acceptance and use of technology). En la cual se reorganizan la restructura del modelo dejando cuatro variables independientes que son la expectativa de funcionamiento, la expectativa de esfuerzo, la influencia social y las condiciones facilitadoras.

Por último, Fathema y Sutton (2013) propusieron un modelo de motivación hedónica del inglés (Hedonic Motivation System Adoption Model HMSAM) en el cual establece como variables independientes la percepción de utilidad, la curiosidad, lo divertido y el control que tiene sobre el sistema. Por lo tanto, después de revisar estos modelos decidimos utilizar las variables que en los estudios empíricos tuvieron mayor significancia y construimos el modelo que se muestra en la Figura 1.

Figura 1. Modelo gráfico propuesto por los autores

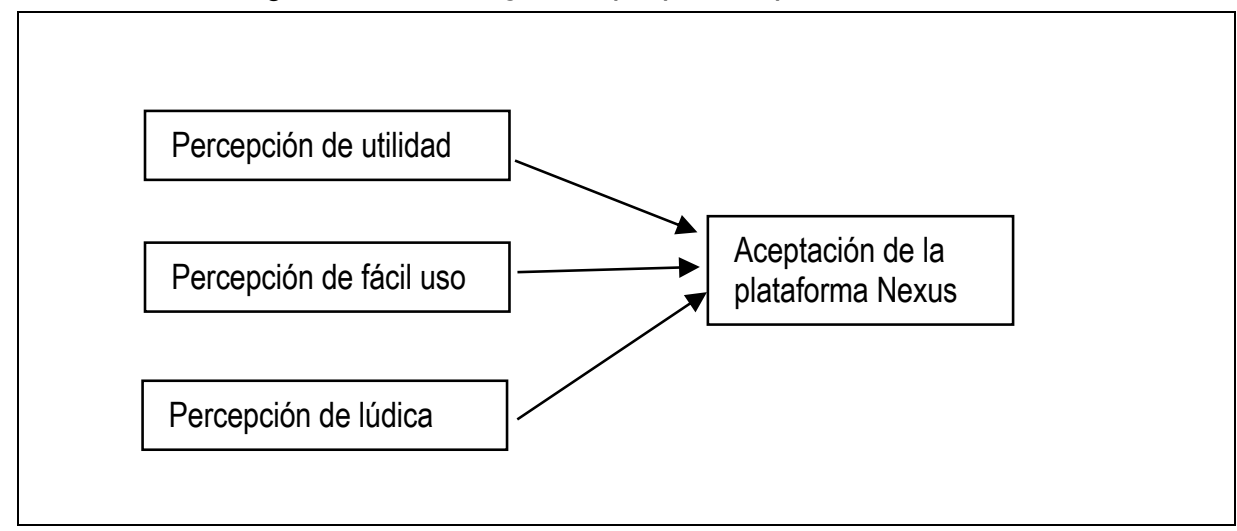

Nota1. Fuente: Elaborado propia.

Todas estas teorías y modelos tienen un objetivo en común conocer los factores que influyen en la aceptación de una herramienta tecnológica. Según Horn, Rothe, \& Gersch (2014) la variable aceptación se define como: una actitud positiva hacia un sistema de información, en términos de la satisfacción acompañada de una intención conductual que a su vez determina el uso real como criterio de aceptación final. . En concordancia con este autor, en este estudio se define la aceptación de la plataforma de E-A Nexus como la satisfacción de haber usado el Nexus, así como el comportamiento que tiene el usuario hacia la utilización real y la intención de usar Nexus en el futuro. 
En cuanto a las variables independientes, percepción de fácil uso y percepción de utilidad, han estado presentes en estudios como los de (AbuShanab, 2017; Cheema, Rizwan, Jalal, Durrani y Sohail, 2013; Lin, Lu, y Liu, 2013; Nistor, Göğüş y Lerche, 2013; Ongena, van de Wijngaert, y Huizer, 2013; Rejón, Liébana y Martínez, 2011; Romero, de Amo y Borja, 2011; Sánchez y Hueros, 2010; Tan, 2013; Tarhini, Hone y Liu, 2013; Wu y Chen, 2017; Zhao y Zhu, 2009) entre otros, comprobando de manera empírica la relación entre estas dos variables y la percepción de aceptación .

Llama la atención que, en todos los estudios mencionados, la variable percepción de utilidad, siempre tuvo una relación más fuerte que la variable percepción de fácil uso y que en ninguno de los estudios se rechazó la hipótesis de su relación.

En estos artículos la definición de utilidad percibida fue la misma que uso Davis (1989), que es el grado en que una persona cree que utilizar un sistema en particular, mejoraría su desempeño laboral y puesto que en este estudio la plataforma que se va a medir es Nexus, la percepción de utilidad se define, como el grado en que un estudiante cree que utilizar Nexus mejoraría su desempeño académico.

En cuanto a la variable percepción de fácil uso, Davis (1989) la define como el grado en que un sistema en particular está libre de esfuerzo, por lo tanto, en este estudio la variable percepción de fácil uso se define, como el grado en que un estudiante cree que la plataforma es intuitiva y usable.

Por otra parte, la variable percepción de lúdica no ha sido encontrada con ese nombre en los estudios empíricos, pero se han encontrado artículos con una variable parecida llamada percepción de disfrute, en modelos como los propuestos por (Cheema et al, 2010; Ongena et al, 2013; Zhao y Zhu, 2009). En estos estudios empíricos, se demostró una relación positiva con la variable dependiente.

Según Chemma et al. (2010) la variable percepción de disfrute, se define como el grado en que una persona disfruta el utilizar un software en particular. Sin embargo, aunque esta variable tiene similitud con la variable percepción de lúdica, la definición es diferente. En este estudio la percepción de lúdica se define como, el grado en que un estudiante disfruta, se divierte y juega mientras utiliza la plataforma. 
De lo anterior se generaron tres hipótesis alternativas:

H1: La utilidad percibida, es un factor positivo en la aceptación de la plataforma Nexus

H2: La percepción de fácil uso, es un factor positivo en la aceptación de la plataforma Nexus

H3: La percepción de lúdica, es un factor positivo en la aceptación de la plataforma Nexus.

\section{Metodología}

El diseño de esta investigación es de tipo cuantitativo, no experimental y de corte transversal. Ya que la forma de recolectar los datos fue a través de una encuesta tipo Likert de 5 opciones, aplicada a estudiantes de la facultad de Filosofía y Letras de manera aleatoria en un tiempo específico. Se utilizó el método de pasos sucesivos de la regresión lineal múltiple, para determinar las variables que tienen una correlación significativa, aunado a esto se verifico que no existiera multicolinealidad entre las variables independientes.

El instrumento de medición consta de 2 partes, en la primera parte se crearon 6 preguntas para medir las variables demográficas y así identificar categorizar a los participantes, en la segunda parte, se crearon 15 preguntas, 5 para cada variable, utilizando una escala de Likert en donde 1 significa totalmente en desacuerdo, 2 en desacuerdo, 3 neutral, 4 de acuerdo y 5 totalmente de acuerdo, estas preguntas se adaptaron de instrumentos utilizados en artículos publicados en revistas de alto impacto, por lo que se considera validez de constructo. La manera de recolección de datos fue a través de la plataforma Google Forms y se envió la liga de la encuesta por correo a cada uno de los participantes.

El contexto en donde se realizó la investigación fue la Facultad de Filosofía y Letras, con los alumnos de noveno y décimo semestre del colegio de ciencias de lenguaje. Se determinó este segmento de la facultad como población, ya que son alumnos que están por egresar y han tenido más experiencia en el uso de la plataforma Nexus que los alumnos de los primeros niveles. En la facultad se imparten 6 cursos en estos niveles con un total de 145 alumnos, a los que se consideran como la población. 
A partir, de esta información se calculó el tamaño de la muestra con la fórmula de la Tabla 2, obteniendo que entrevistar a 102 estudiantes, es suficiente para que la muestra pueda generalizarse, a un nivel de confianza del $95 \%$, y con un error de $5 \%$. En este estudio, se aplicaron 107 encuestas, por lo tanto, se pueden generalizar los resultados a el resto de la población.

Tabla 2. Fórmula para calcular el tamaño de la muestra para población finita

\begin{tabular}{|c|c|c|}
\hline Variable & Valor & Definición \\
\hline$n=$ & 102 & $\mathrm{Ns}^{2} /\left((\mathrm{N}-1)(\mathrm{e} / \mathrm{z})^{2}+\mathrm{s}^{2}\right)$ \\
\hline Media & 3.00 & $\begin{array}{l}\text { Obtenidas a partir del número de ítems de } \\
\text { la escala de Likert }\end{array}$ \\
\hline Varianza & 2.00 & $\begin{array}{l}\text { Obtenidas a partir del número de ítems de } \\
\text { la escala de Likert }\end{array}$ \\
\hline Desviación estándar estimada= & 1.41 & Raíz de la varianza \\
\hline$N=$ & 145 & $\begin{array}{l}\text { Tamaño de la población delimitado por el } \\
\text { autor. }\end{array}$ \\
\hline$e=$ & 0.15 & $\begin{array}{l}\text { Error tolerable en porcentaje estimado } \\
\text { (Media * }{ }^{* 05)}\end{array}$ \\
\hline Confianza= & $95 \%$ & $\begin{array}{l}\text { Expectativa que sea una de los } 95 \text { de / } \\
100 \ldots\end{array}$ \\
\hline Área= & 0.975 & Área acumulada. \\
\hline$Z=$ & 1.960 & Parámetro de gauss equivalente al área \\
\hline
\end{tabular}

Fuente: Elaboración propia.

\section{Resultados}

Se aplicó la prueba piloto a un grupo de 15 estudiantes, obteniendo un Alfa de Cronbach superior a .8 en cada uno de los constructos. En la Tabla 3 se muestran los resultados del Alpha de Cronbach, obtenidos mediante el software SPSS. Cabe mencionar que el software sugirió, eliminar algunos ítems para aumentar más la confiabilidad, sin embargo, se decidió no eliminar ninguno de ellos para tener un poco más de variabilidad en las sumatorias por constructo. 
Tabla 3. Confiabilidad del instrumento

\begin{tabular}{lc}
\hline Constructo & Alfa de Cronbach \\
\hline Percepción de Utilidad & .873 \\
Percepción de Fácil Uso & .864 \\
Percepción de Lúdica & .870 \\
Percepción de Aceptación & .884 \\
\hline
\end{tabular}

Fuente: Elaboración propia.

Continuando con el análisis, se obtuvo la media ponderada de los ítems de cada constructo, utilizando como ponderador la escala de correlación obtenida mediante el SPSS, en la opción de "Escala si se elimina el elemento". Después se hicieron 7 regresiones lineales, utilizando el método de pasos sucesivos en el software SPSS. En las primeras tres regresiones se dio entrada a una sola variable independiente, es decir, fueron regresiones simples. De la regresión numero 4 a la 6 se dieron entrada a dos variables independientes y por último en la séptima regresión se dio de entrada a las 3 variables independientes al mismo tiempo. Los resultados obtenidos se pueden observar en la Tabla 4.

Tabla 4. Análisis de regresión lineal por el método de pasos sucesivos

\begin{tabular}{lcl}
\hline Variables del modelo & R cuadrada & Nivel de significancia \\
\hline Percepción de Utilidad (PU) & .798 & .000 \\
Percepción de Lúdica (PL) & .884 & .000 \\
Percepción de Fácil Uso (PFU) & .693 & .000 \\
PU y PL & .903 & PU .000, PL .000 \\
PU y PFU & .843 & PU .000, PFU .000 \\
PL y PFU & .893 & PL .000, PFU .007 \\
PU, PL y PFU & .908 & PU .000, PL .000, PFU .024 \\
\hline
\end{tabular}

Fuente: Elaboración propia.

Se decidió, obtener 7 regresiones lineales, para comparar las diferencias entre las $\mathrm{R}$ cuadradas de cada uno de los modelos, y determinar el nivel de explicación de cada modelo. Como se observa en la tabla 4, el factor más importante para la aceptación de la plataforma es la percepción de 
lúdica, seguido de la percepción de utilidad, y por último la percepción de fácil uso. Las tres variables tuvieron un nivel de significancia menor a .05 , por lo que se considera que ninguna de las tres hipótesis se rechaza; en este mismo sentido, se puede observar que el nivel de explicación del modelo aumenta al incluir más variables, siendo la regresión múltiple que involucra a las tres variables, la regresión con la $\mathrm{r}^{2}$ cuadrada más alta.

Al observar los resultados de la Tabla 4, se pudiera pensar en un nivel de multicolinealidad entre las variables independientes, para descartar lo anterior, se procedió a calcular la correlación entre todos los constructos. La Tabla 5 muestra las correlaciones entre todas las variables del modelo.

Tabla 5.- Matriz de correlación entre las variables del modelo

\begin{tabular}{lllll}
\hline Variable & PA & PU & PFU & PL \\
\hline PA & 1.000 & 0.636 & 0.48 & 0.781 \\
PU & 0.636 & 1.000 & 0.354 & 0.591 \\
PFU & 0.480 & 0.354 & 1.000 & 0.461 \\
PL & 0.781 & 0.591 & 0.461 & 1.000 \\
\hline
\end{tabular}

Fuente: Elaboración propia.

En la Tabla 5, se puede apreciar una correlación de .591 entre las variables percepción de utilidad (PU) y percepción de lúdica $(\mathrm{PL})$, lo que muestra una relación débil entre estas dos variables independientes. Por otro lado, la relación entre las variables percepción de lúdica y percepción de fácil uso (PFU) muestra una relación de .461 que se puede considerar también como una relación débil y por ultimo la correlación entre las variables percepción de utilidad (PU) y percepción de fácil uso (PFU) muestran una correlación de .354 la correlación más débil de todas.

Por lo anterior, se procedió a realizar un diagnóstico de colinealidad en el SPSS para corroborar si existe colinealidad entre las variables independientes sobre todo entre las variables percepción de utilidad y percepción de lúdica que son las que tiene la correlación más fuerte, los resultados se muestran en la Tabla 6. 
Tabla 6. Diagnóstico de colinealidad

\begin{tabular}{cccccc}
\hline \multirow{2}{*}{ Autovalores } & Índice de & \multicolumn{5}{c}{ Proporciones de la varianza } \\
& condición & (Constante) & PLP & UPP & PFUP \\
\hline 3.893 & 1.000 & .00 & .00 & .00 & .00 \\
0.059 & 8.104 & .58 & .19 & .04 & .00 \\
0.029 & 11.685 & .13 & .00 & .39 & .73 \\
0.019 & 14.243 & .29 & .80 & .56 & .27 \\
\hline
\end{tabular}

Fuente:(Tabla creada por el autor)

Según Belsley (1991), el índice de condición indica el grado de multicolinealidad entre las variables independientes, para él, un índice de condición menor a 30, indica que el nivel de colinealidad entre las variables es bajo 0 nulo, por lo anterior, podemos decir que no existe alta colinealidad, entre las variables independientes del modelo propuesto.

\section{Conclusiones}

Después de analizar los resultados, se concluye que los factores más importantes que influyen en la aceptación de la plataforma de enseñanzaaprendizaje Nexus, son la percepción de lúdica, la percepción de utilidad y la percepción de fácil uso, ya que ninguna de las hipótesis fue rechazada. Uno de los principales hallazgos de este estudio, fue que la percepción de lúdica tuvo una correlación mayor, que las variables percepción de utilidad y percepción de fácil uso, a pesar de que en la revisión de literatura el modelo TAM, no incluye dicha variable.

Otros hallazgos importantes fueron, el alto grado de explicación que tiene este modelo, la parsimonia del modelo, que no muestra colinealidad entre las variables independientes y que puede ser extrapolado, para medir diferentes herramientas de la tecnología educativa.

Para futuras investigaciones, se tiene contemplado realizar un estudio que abarque más facultades de la UANL, de otras áreas del conocimiento, como las ciencias sociales, las ciencias exactas y las ciencias de la salud. Por otro lado, se planea agregar al final de la encuesta, un cuestionario de estilos de aprendizaje, esto con el objetivo de realizar comparaciones entre alumnos con distintos estilos de aprendizaje. También se tiene considerado realizar un estudio cualitativo de tipo fenomenológico, que permita complementar este 
estudio con un enfoque mixto de tipo secuencial, triangulando los resultados obtenidos.

Por último, se tiene considerado compartir, los hallazgos de este estudio con la Dirección de Tecnologías de Información de la UANL, para que tomen mejores decisiones en cuanto a las estrategias para mejorar las variables independientes; y esto a su vez mejore la aceptación del Nexus. Lo anterior, con el fin de realizar estudios longitudinales, que nos permitan saber si las estrategias que se están implementado están dando resultados.

Como conclusión general, el modelo TAM fue diseñado con el fin de medir la aceptación de tecnología en las empresas y se orientó a empleados que utilizan tecnología en sus actividades diarias, sin embargo, en el ámbito educativo, la tecnología está dirigida a estudiantes los cuales pertenecen a una generación en donde el hedonismo juega un papel muy importante; los resultados comprueban que el modelo de aceptación de tecnología propuesto, en este estudio es más indicado para el ámbito educativo.

\section{Referencias}

Abu-Shanab, E. A. (2017). E-government familiarity influence on Jordanians' perceptions, Telematics and Informatics, 34(1), 103-113.

Ajzen, I. (1985). From intentions to actions: A theory of planned behavior. En J. Kuhl, \& J. Beckman (Eds.), Action-control: From cognition to behavior (pp. 11-39). Heidelberg: Springer.

Ajzen, I. \& Fishbein, M. (1980). Understanding attitudes and predicting social behavior. Englewood Cliffs, NJ: Prentice.

ANUIES (2017). Anuario de educación superior 2016-2017. Ciudsd de México: Asociación Nacional de Universidades e Instituciones de Educación Superior.

Belsley, D. A. (1991). Conditioning diagnostics: Collinearity and weak data in regression. New York: Wiley.

Cheema, U., Rizwan, M., Jalal, R., Durrani, F. \& Sohail, N. (2013). The trend of online shopping in 21st century: Impact of enjoyment in TAM model, Asian Journal of Empirical Research, 3(2), 131-141.

Davis, F. D. (1989). Perceived usefulness, perceived ease of use, and user acceptance of information technology, MIS Quarterly, 13(3), 319-340. 
Fathema, N. \& Sutton, K. L. (2013). Factors influencing faculty members' Learning Management Systems adoption behavior: An analysis using the Technology Acceptance Model, International Journal of Trends in Economics Management \& Technology (IJTEMT), 2(6), 20-28.

Horn, A. M., Rothe, H. \& Gersch, M. (2014). Which factors drive e-learning usage?. Trabajo presentado en INTED 2014 Conference, marzo de 2014, Valencia, España.

Lin, P. C., Lu, H. K. \& Liu, S. C. (2013). Towards an education behavioral intention model for e-learning systems: an extension of UTAUT, Journal of Theoretical \& Applied Information Technology, 47(3), 1120-1127.

Marangunić, N. \& Granić, A. (2015). Technology acceptance model: a literature review from 1986 to 2013, Universal Access in the Information Society, 14(1), 81-95.

Nistor, N., Göğüş, A. \& Lerche, T. (2013). Educational technology acceptance across national and professional cultures: a European study, Educational Technology Research and Development, 61(4), 733-749.

Ongena, G., van de Wijngaert, L. \& Huizer, E. (2013). Acceptance of online audio-visual cultural heritage archive services: a study of the general public, Information research, 18(2), 1-17.

Rejón, F., Liébana, F. J. \& Martínez, M. (2011). Factores motivacionales de la aceptación de redes sociales de microblogging: modelo $\mu$ btam. Trabajo presentado en el Tercer congreso internacional UNIVEST, junio de 2011, Cataluña, España.

Romero, C. L., de Amo, M. D. C. A. \& Borja, M. Á. G. (2011). Adopción de redes sociales virtuales: ampliación del modelo de aceptación tecnológica integrando confianza y riesgo percibido, Cuadernos de Economía y Dirección de la Empresa, 14(3), 194-205.

Sánchez, R. A. \& Hueros, A. D. (2010). Motivational factors that influence the acceptance of Moodle using TAM, Computers in human behavior, 26(6), 1632-1640.

Tan, P. J. B. (2013). Applying the UTAUT to understand factors affecting the use of English elearning websites in Taiwan, Sage Open, 3(4), 1-12.

Tarhini, A., Hone, K. \& Liu, X. (2013). User acceptance towards web-based learning systems: Investigating the role of social, organizational and individual factors in European higher education, Procedia Computer Science, 17, 189-197.

Venkatesh, V., \& Davis, F. D. (2000). A theoretical extension of the technology acceptance model: Four longitudinal field studies. Management science, 46(2), 186-204.

Venkateh, V., Morris, M., Davis, G., \& Davis, F. (2003). User acceptance of information technology: toward a unified view, MIS Quarterly, 27(3), 425-478.

Wu, B., \& Chen, X. (2017). Continuance intention to use MOOCs: Integrating the technology acceptance model (TAM) and task technology fit (TTF) model, Computers in Human Behavior, 67, 221-232.

Zhao, Y., \& Zhu, Q. (2009). Blog acceptance model: An empirical study on exploring users' acceptance and continual usage of blogs, Journal of Data and Information Science, 2(3), 44-61. 